\title{
Determination of the $\mathrm{N}-\mathrm{H}$ Bond Dissociation Free Energy in a Pyridine(diimine) Molybdenum Complex Prepared by Proton Coupled Electron Transfer
}

\author{
Grant W. Margulieux, Sangmin Kim, and Paul J. Chirik* \\ Department of Chemistry, Princeton University, Princeton, New Jersey 08544, USA.
}

\section{Table of Contents}

1. NMR Spectroscopic Data

2. Cyclic Voltammograms

S4

3. DFT Input File Examples

S6

4. Coordinates from the Geometry Optimizations

S8 


\section{NMR Spectroscopic Data}

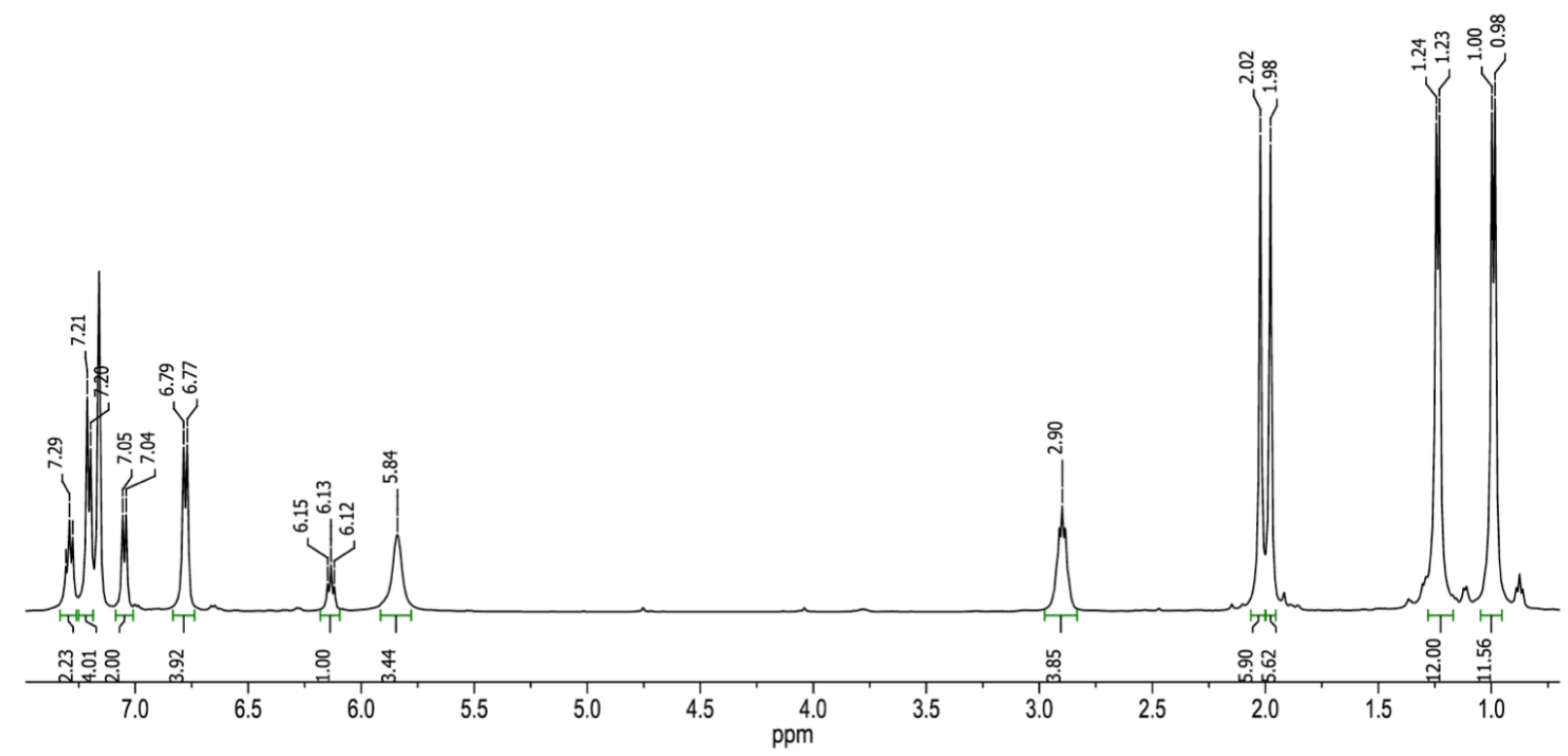

Figure S1. Representative ${ }^{1} \mathrm{H}$ NMR spectrum of $\mathbf{2}$ in benzene- $d_{6}$.

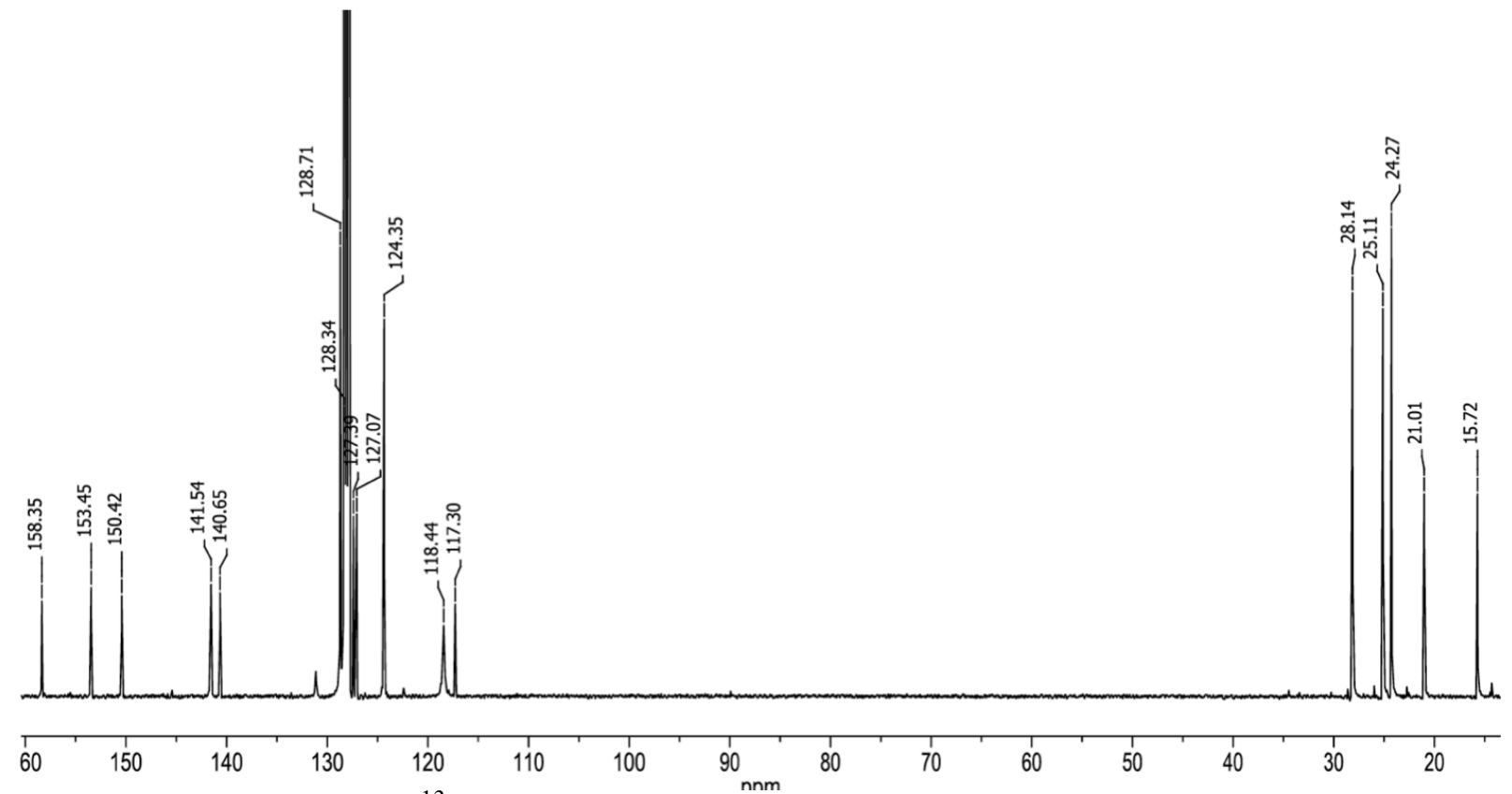

Figure S2. Representative ${ }^{13} \mathrm{C}$ NMR spectrum of 2 in benzene- $d_{6}$. 


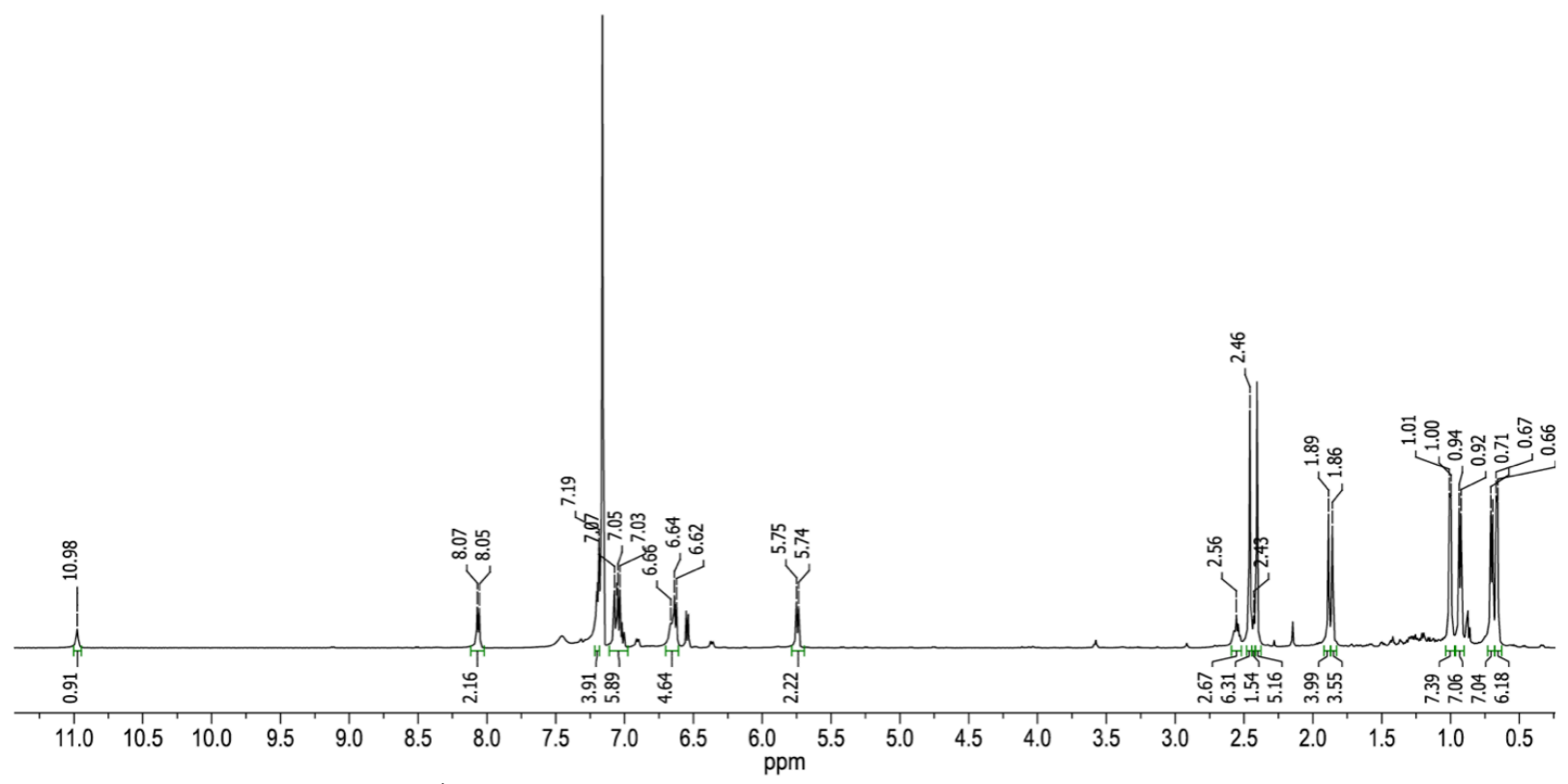

Figure S3. Representative ${ }^{1} \mathrm{H}$ NMR spectrum of 3-OTf in benzene- $d_{6}$.

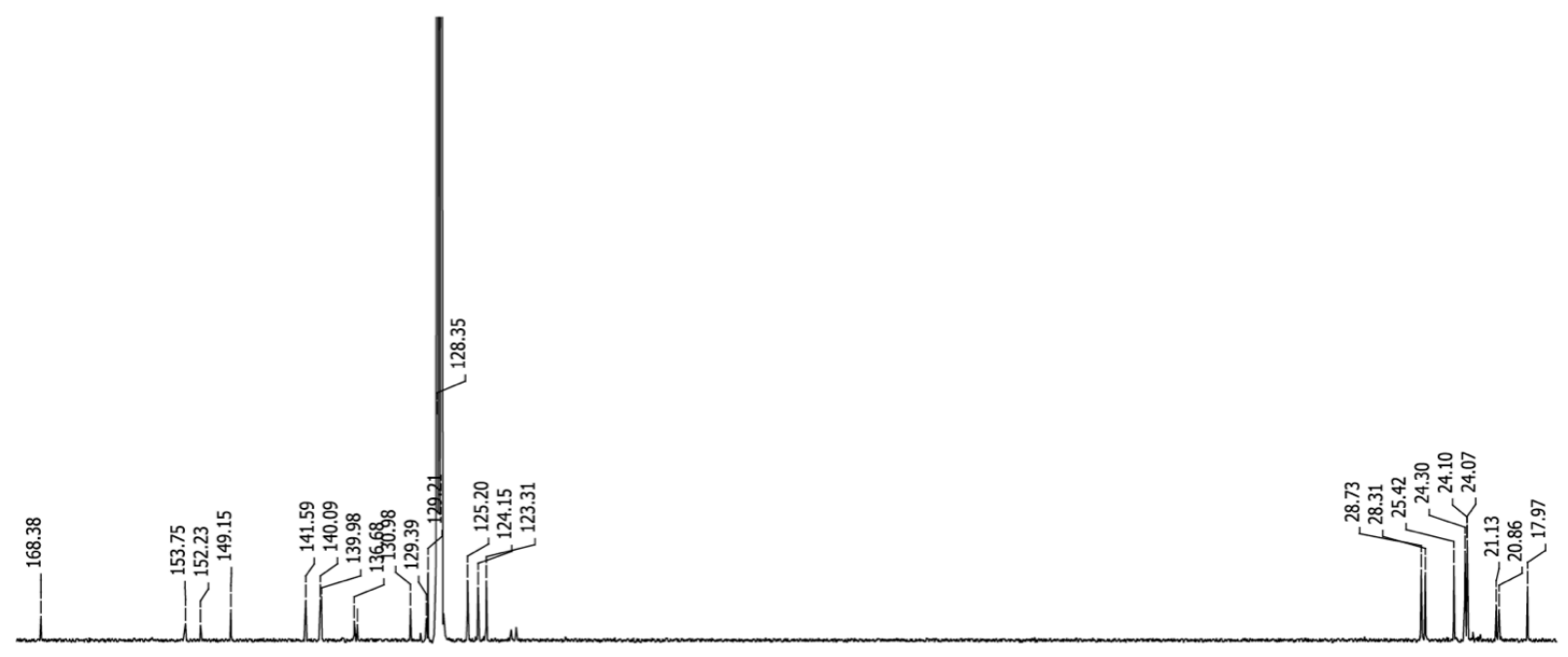

\begin{tabular}{|c|}
\hline 16 \\
\hline
\end{tabular}

Figure S4. Representative ${ }^{13} \mathrm{C}$ NMR spectrum of 3-OTf in benzene- $d_{6}$. 


\section{Cyclic Voltammograms}

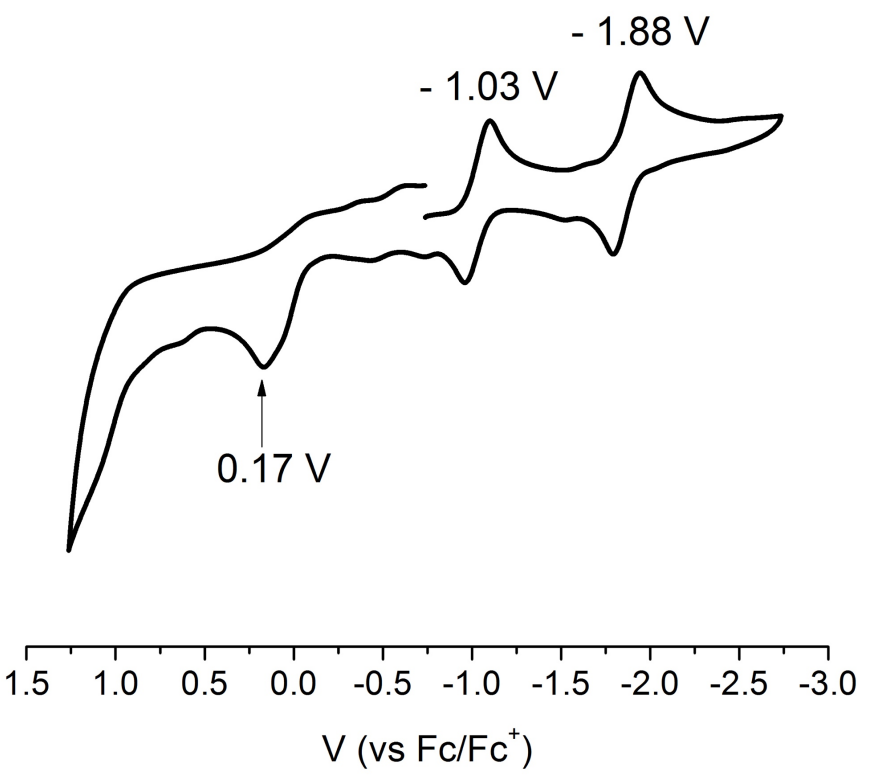

Figure S5. Cyclic voltammogram of 3-OTf using a glassy-carbon working electrode, a platinum wire counter electrode, a silver wire reference electrode, $0.1 \mathrm{M}\left[{ }^{\mathrm{n}} \mathrm{Bu}_{4} \mathrm{~N}\right]\left[\mathrm{PF}_{6}\right]$, and a scan rate of $100 \mathrm{mV} / \mathrm{s}$ in $\mathrm{THF}$ at $295 \mathrm{~K}$ versus $\mathrm{Cp}_{2} \mathrm{Fe} / \mathrm{Cp}_{2} \mathrm{Fe}^{+}$.
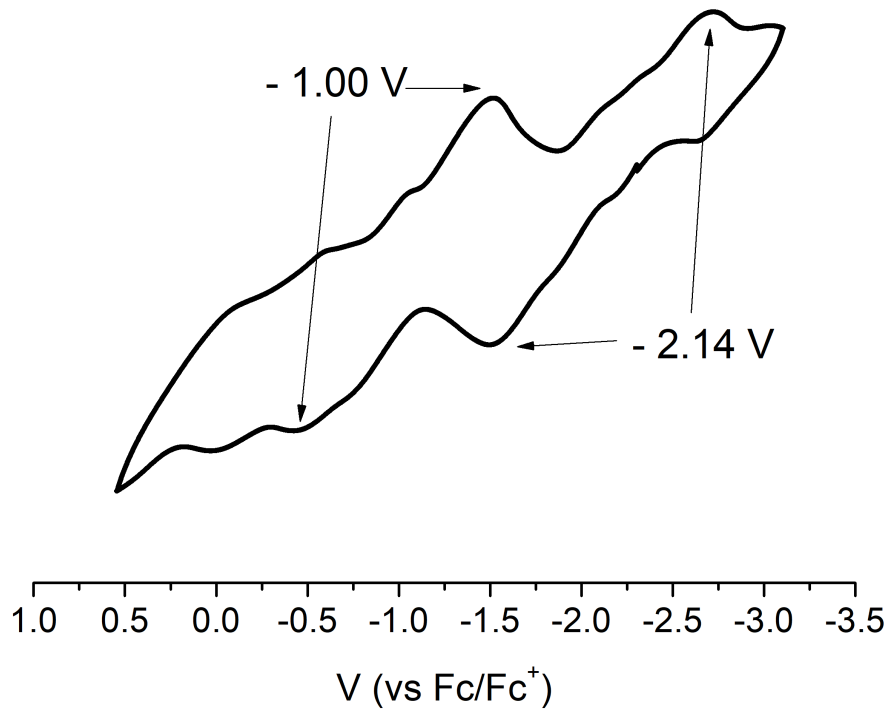

Figure S6. Cyclic voltammogram of K-5 using a glassy-carbon working electrode, a platinum wire counter electrode, a silver wire reference electrode, $0.1 \mathrm{M}\left[{ }^{\mathrm{n}} \mathrm{Bu}_{4} \mathrm{~N}\right]\left[\mathrm{PF}_{6}\right]$, an $\mathrm{d}$ a scan rate of $100 \mathrm{mV} / \mathrm{s}$ in $\mathrm{THF}$ at $295 \mathrm{~K}$ versus $\mathrm{Cp}_{2} \mathrm{Fe} / \mathrm{Cp}_{2} \mathrm{Fe}^{+}$. 


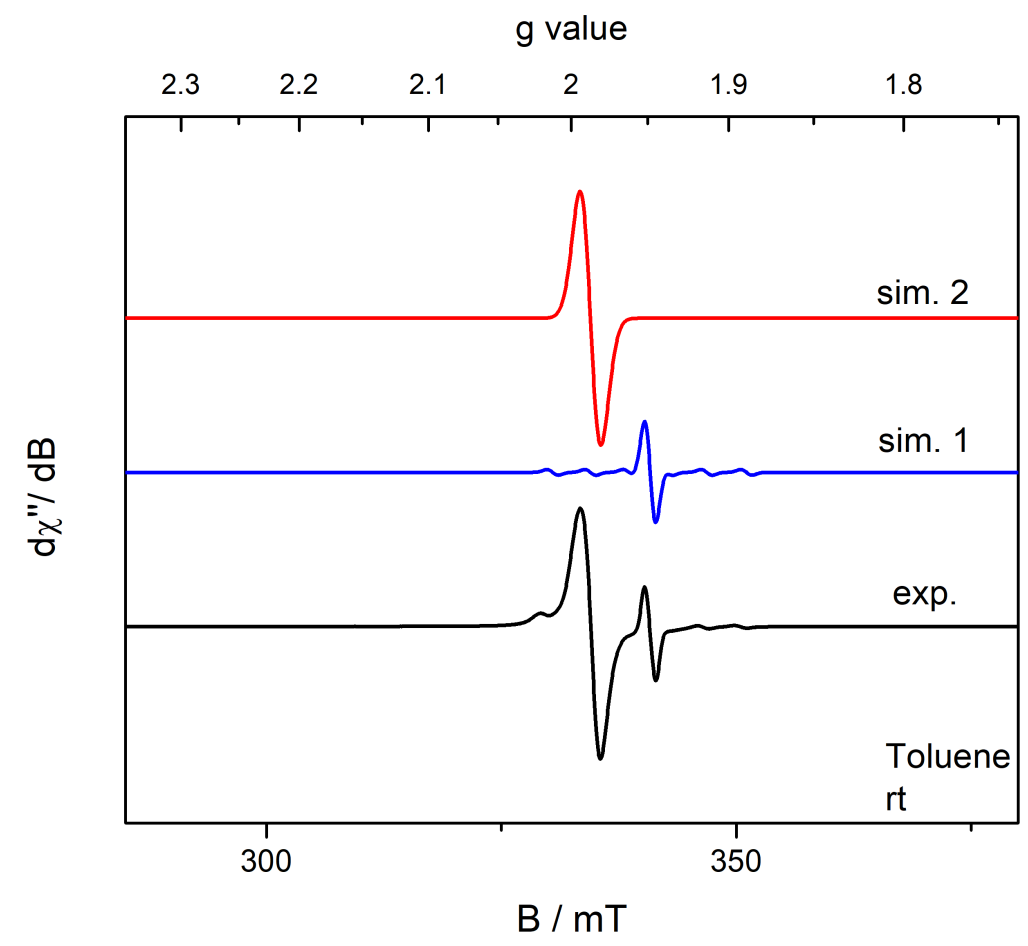

Figure S7. Representative X-band EPR spectrum of the crude mixture of $\mathbf{K - 5}$ and cyclopentadiene recorded at room temperature in toluene for $\mathrm{p} K_{\mathrm{a}}$ measurements (black). Simulation gave two isotropic signals which were assigned to $\mathbf{4}$ (blue) and K-5 (red), respectively.

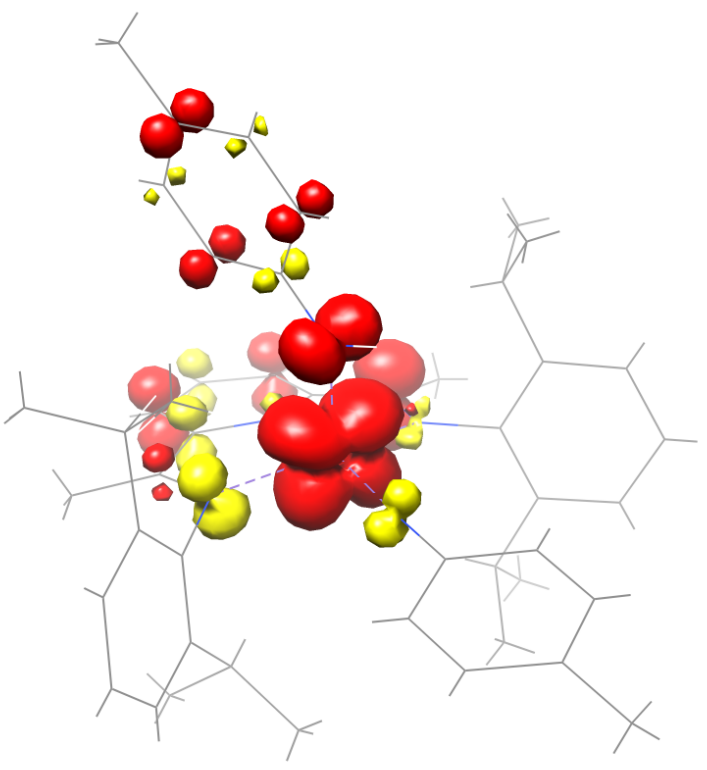

Figure S8. DFT-computed SOMO of 4. 


\section{DFT Input File Examples \\ 3.1 Geometry Optimiations}

$\left({ }^{\mathrm{Pr} P D I}\right) \mathrm{Mo}(=\mathrm{NTol})_{2}(\mathrm{~S}=\mathbf{0})$

! RKS B3LYP RIJCOSX ZORA ZORA-def2-SVP SARC/J Normalprint SlowConv TightSCF Opt Pal8 UCO

\%basis NewGTO 42 "old-ZORA-TZVP" end

NewGTO 7 "ZORA-DEF2-TZVP(-f)" end

NewAuxGTO 42 "SARC/J" end

NewAuxGTO 7 "SARC/J" end

end

\%SCF MaxIter 500

TolE 1e-7

TolErr 1e-6

end

* xyz 01

XYZ Coordinates

$*$

$\left({ }^{i P r} \mathrm{PDI}\right) \mathrm{Mo}(\mathrm{NHTol})(=\mathrm{NTol})(\mathrm{S}=1 / 2)$

! UKS B3LYP RIJCOSX ZORA ZORA-def2-SVP SARC/J Normalprint SlowConv TightSCF Opt Pal8 UCO

\%basis NewGTO 42 "old-ZORA-TZVP" end

NewGTO 7 "ZORA-DEF2-TZVP(-f)" end

NewAuxGTO 42 "SARC/J" end

NewAuxGTO 7 "SARC/J" end

end

\%SCF MaxIter 500

TolE 1e-7

TolErr 1e-6

end

* xyz 02

XYZ Coordinates

$*$

3.2 Numerical Frequency Calculations

$\left({ }^{i P r} \mathrm{PDI}\right) \mathrm{Mo}(=\mathrm{NTol})_{2}(\mathrm{~S}=\mathbf{0})$

! RKS B3LYP RIJCOSX SlowConv TightSCF ZORA ZORA-def2-SVP SARC/J Normalprint Numfreq Grid4 Nofinalgrid Pal8 
\%basis NewGTO 42 "old-ZORA-TZVP" end NewGTO 7 "ZORA-DEF2-TZVP(-f)" end

NewAuxGTO 42 "SARC/J" end

NewAuxGTO 7 "SARC/J" end

end

\%SCF MaxIter 500

TolE 1e-7

TolErr 1e-6

end

\%FREQ RESTART TRUE

CENTRALDIFF TRUE

INCREMENT 0.01

END

* xyz 01

XYZ Coordinates

*

$\left.{ }^{i P r} P D I\right) M o(N H T o l)(=N T o l)(S=1 / 2)$

! UKS B3LYP RIJCOSX SlowConv TightSCF ZORA ZORA-def2-SVP SARC/J Normalprint Numfreq Grid4 Nofinalgrid Pal8

\%basis NewGTO 42 "old-ZORA-TZVP" end NewGTO 7 "ZORA-DEF2-TZVP(-f)" end

NewAuxGTO 42 "SARC/J" end

NewAuxGTO 7 "SARC/J" end

end

\%SCF MaxIter 500

TolE $1 \mathrm{e}-7$

TolErr 1e-6

end

\%FREQ RESTART TRUE

CENTRALDIFF TRUE

INCREMENT 0.01

END

* xyz 02

XYZ Coordinates

* 


\begin{tabular}{|c|c|c|c|}
\hline \multicolumn{4}{|c|}{$\begin{array}{l}\text { 4. Coordinates from Geometry Optimizations } \\
\left({ }^{P r} \mathrm{PDI}\right) \mathrm{Mo}(=\mathrm{NTol})_{2}(\mathrm{~S}=\mathrm{0})\end{array}$} \\
\hline $\mathrm{C}$ & 0.55168604445613 & 4.19889812584769 & 1.30157620208619 \\
\hline $\mathrm{H}$ & 1.64213258507727 & 4.33798055477845 & 25979233 \\
\hline $\mathrm{H}$ & 0.18070185665537 & & 2.21999598621270 \\
\hline $\mathrm{H}$ & 0.13446175948838 & 4.74171665892446 & 0.45055233175503 \\
\hline $\mathrm{C}$ & 0.19083679393953 & & 2471 \\
\hline $\mathrm{C}$ & 0.32049980413063 & 1.89217663429293 & 6841 \\
\hline $\mathrm{C}$ & & & 4821 \\
\hline $\mathrm{H}$ & 407893545779 & & 3272 \\
\hline $\mathrm{C}$ & 46300792 & & \\
\hline $\mathrm{H}$ & 0.97611029401558 & & 927 \\
\hline $\mathrm{C}$ & 0.366286141066 & & \\
\hline $\mathrm{H}$ & 0.35 & & \\
\hline $\mathrm{C}$ & 0.002837 & -0.3 & \\
\hline $\mathrm{C}$ & -0.52487 & & \\
\hline $\mathrm{C}$ & -0.56 & 572 & \\
\hline $\mathrm{H}$ & & & \\
\hline $\mathrm{H}$ & -0.2 & 536 & \\
\hline $\mathrm{H}$ & & & \\
\hline $\mathrm{C}$ & & & \\
\hline $\mathrm{C}$ & & & \\
\hline $\mathrm{C}$ & & & \\
\hline $\mathrm{H}$ & -5.22215 & & \\
\hline $\mathrm{C}$ & & & \\
\hline $\mathrm{H}$ & -4.43066 & 005 & \\
\hline $\mathrm{C}$ & & & \\
\hline $\mathrm{H}$ & -2.01 & 293 & 041 \\
\hline $\mathrm{C}$ & & & \\
\hline $\mathrm{C}$ & 0.06 & & \\
\hline $\mathrm{H}$ & 0.654 & & \\
\hline $\mathrm{C}$ & 0.34 & & \\
\hline $\mathrm{H}$ & -0.22797 & & \\
\hline $\mathrm{H}$ & & & 837 \\
\hline $\mathrm{H}$ & 0.078 & & \\
\hline $\mathrm{C}$ & 0.52 & -5.4 & \\
\hline $\mathrm{H}$ & 0.34 & & \\
\hline $\mathrm{H}$ & 1.60463 & & \\
\hline $\mathrm{H}$ & 0.01065363488296 & & \\
\hline $\mathrm{C}$ & -3.80722349891087 & & \\
\hline $\mathrm{H}$ & -2.99187998695365 & 49526 & 2887380 \\
\hline $\mathrm{C}$ & -4.24914374790268 & & \\
\hline $\mathrm{H}$ & -5.06595285065024 & -2.33076872693763 & 3.46627523008856 \\
\hline $\mathrm{H}$ & -4.61187061451061 & & \\
\hline $\mathrm{H}$ & -3.42828235198072 & -2.01520036570280 & 4.06016043579934 \\
\hline $\mathrm{C}$ & -4.95649941535103 & -0.57639925798986 & 1.26093743376625 \\
\hline
\end{tabular}




\begin{tabular}{|c|c|c|c|}
\hline & -4.65320223757815 & -0.32454241001879 & \\
\hline & -5.24888255730056 & 0.35387417578805 & 76623627428172 \\
\hline & -5.84928617727762 & -1.21307446977086 & 1.20767837983342 \\
\hline & -0.49911816753376 & 2.85819234501201 & \\
\hline & -1.81278181119528 & 3.33396875327407 & \\
\hline & -2.04781920946715 & & \\
\hline & -3.04678430781596 & 4.40190546360131 & -2.714 \\
\hline & -1.02979102122584 & 4.24968450107016 & -3.4280344818 \\
\hline & -1.23917130658461 & 4.78963766918884 & -4.351010735 \\
\hline & 0.25643233628109 & 3.7879717 & -3.158 \\
\hline & 1.053 & & \\
\hline & 0.552325200 & 3.08 & -1.9758 \\
\hline & & & \\
\hline & 2.005 & 2.06 & -0.7966 \\
\hline & 2.943 & & \\
\hline & 3.059039 & 4.401 & -2.483 \\
\hline & 3.93886797 & & -1.2456 \\
\hline & 2.586 & & \\
\hline & 2.508 & & -2.84 \\
\hline & 1.84 & & \\
\hline & 3.50 & & -2.584 \\
\hline & 2.58 & 2.24 & -3.800 \\
\hline & -2.94 & & -0.29 \\
\hline & -2.62 & & \\
\hline & -3.21 & & \\
\hline & -3.58 & 5.26 & -0.20 \\
\hline & -2.30 & & \\
\hline & -3.96 & & \\
\hline & -4.25 & & \\
\hline & -4.67 & & -1.6 \\
\hline & -5.00 & 2.47 & -0.158 \\
\hline & -4.08 & 1.72 & -1.482 \\
\hline & & -0.6 & \\
\hline & -1.43 & -0.0 & -3.58 \\
\hline & -0.6 & & -3.48 \\
\hline & -1.91 & -0.4 & -4.837 \\
\hline & -1.44 & -0.0 & -5.721 \\
\hline & -2.96995338010003 & -1.368 & -4.99464500 \\
\hline & -3.45 & -1.77 & \\
\hline & -4.25725351569219 & -2.521 & -6.30419458 \\
\hline & -2.64723227074624 & -2.207 & -6.97328886 \\
\hline $\mathrm{H}$ & -3.85297291682711 & -0.915 & -6.92960896877639 \\
\hline & -3.54808346796014 & -1.89126480229802 & -3.82649851114056 \\
\hline & -4.36830424216582 & -2.60584851877969 & \\
\hline & -3.11133736052328 & -1.51211027201998 & -2.55602878428709 \\
\hline & -3.57837696990938 & -1.92957625669351 & -1.66688050688424 \\
\hline
\end{tabular}




$\begin{array}{llcc}\text { C } & 2.66473628519907 & -0.68021291950652 & -0.24540018563377 \\ \text { C } & 3.43974157506222 & -1.42226982781829 & -1.16914848012771 \\ \text { H } & 2.92893890840894 & -1.93578783293597 & -1.98145166142197 \\ \text { C } & 4.82795101558390 & -1.47377059421880 & -1.05431710686986 \\ \text { H } & 5.40120493978633 & -2.04671326791341 & -1.78499384754925 \\ \text { C } & 5.50791480381195 & -0.78557367488361 & -0.03064847190354 \\ \text { C } & 4.73640128835131 & -0.05612999420109 & 0.88964360740719 \\ \text { H } & 5.23822202527160 & 0.48385888660980 & 1.69421123532884 \\ \text { C } & 3.34588329543233 & -0.00761179881987 & 0.79782222062774 \\ \text { H } & 2.76223301171079 & 0.55769395709278 & 1.52111183215610 \\ \text { C } & 7.01366550395331 & -0.83233345387608 & 0.07876245422680 \\ \text { H } & 7.36556862339115 & -1.83011862738290 & 0.38026070423538 \\ \text { H } & 7.38239782005660 & -0.11671032978611 & 0.82205374263818 \\ \text { H } & 7.49548077769773 & -0.59575667453713 & -0.87996722960689 \\ \text { N } & -0.26094649691289 & 2.11371322717156 & 0.16243453066401 \\ \text { N } & 0.07432230357514 & 0.55622668178113 & 2.11679560813501 \\ \text { N } & -0.98012499922316 & -1.62573404687257 & 1.47080131431890 \\ \text { N } & -1.55412422648071 & -0.28979595177350 & -1.16245376855621 \\ \text { N } & 1.30016775979732 & -0.57194670167328 & -0.38818349304170 \\ \text { Mo } & -0.33144186809878 & -0.07464453727536 & 0.15530087833823\end{array}$

\begin{tabular}{|c|c|c|c|}
\hline \multicolumn{4}{|c|}{$\left.{ }^{i P r} P D I\right) M o(N H T o l)(=N T o l)(S=1 / 2)$} \\
\hline $\mathrm{C}$ & 3.95475867344229 & -2.40791956660158 & -0.85944896 \\
\hline $\mathrm{H}$ & 3.99396132728207 & & \\
\hline $\mathrm{H}$ & 4.35167143684007 & -2.78981033407676 & \\
\hline $\mathrm{H}$ & 63371228320960 & & 52682 \\
\hline $\mathrm{C}$ & 55172812040122 & -1.88943457791942 & 6474 \\
\hline $\mathrm{C}$ & 93028 & -2.74 & 331 \\
\hline $\mathrm{C}$ & 531 & $-4.1<$ & \\
\hline $\mathrm{H}$ & 10647 & -4.6 & 521 \\
\hline $\mathrm{C}$ & & -4.8 & \\
\hline $\mathrm{H}$ & 53 & -5.9 & -0 . \\
\hline $\mathrm{C}$ & -0.98 & & \\
\hline $\mathrm{H}$ & 58142 & -4 & 045 \\
\hline $\mathrm{C}$ & -0.9 & & \\
\hline $\mathrm{C}$ & -2.08 & & 058 \\
\hline $\mathrm{C}$ & 318458 & -2.3 & \\
\hline $\mathrm{H}$ & -3.77 & & 400 \\
\hline $\mathrm{H}$ & -3.55 & -3.0 & \\
\hline $\mathrm{H}$ & -4.21 & -1.5 & 2962 \\
\hline $\mathrm{C}$ & -2.83 & & \\
\hline $\mathrm{C}$ & 64091097 & 0.99 & 0727 \\
\hline $\mathrm{C}$ & -4.23056383626188 & & 97468 \\
\hline $\mathrm{H}$ & -4.49844038416453 & & -2.77824 \\
\hline $\mathrm{C}$ & -4.97352259235691 & 2.21681969100256 & -0.68788176256643 \\
\hline $\mathrm{H}$ & -5.81077070476310 & 2.90954906259168 & -0.7701168814326 \\
\hline
\end{tabular}




\begin{tabular}{|c|c|c|c|}
\hline & -4.63527474365095 & 1.66357766169899 & \\
\hline & -5.21386184058908 & 1.93178116102040 & 1.42858419128936 \\
\hline & -3.57591032748114 & 0.75154965093299 & 0.67441424299604 \\
\hline & -3.27522600931257 & 0.15515134247578 & 2.04651620040871 \\
\hline & -2.47477253705113 & -0.57957898504570 & \\
\hline & -4.48389332510849 & -0.58562917906299 & 2.65077 \\
\hline & -5.29551021328475 & 0.10541773976527 & \\
\hline & -4.17982652424847 & -1.10619336888075 & \\
\hline & -4.89506888426393 & 54550686664 & \\
\hline & -2.75287024273924 & 01992842645 & \\
\hline & 0185914 & 59111 & \\
\hline & -2.4374316 & 0.75649857018182 & \\
\hline & -3.52514153768434 & & \\
\hline$y$ & -2.3747600 & 0.6564 & -3.02 \\
\hline & -1.4592454 & 963016053 & -2.7 \\
\hline$y$ & -1.94762 & & \\
\hline & -2.81122 & 2.45 & 32434 \\
\hline & -1.30 & & \\
\hline $\mathrm{H}$ & -1.38954 & 2.610 & -3.1 \\
\hline & -3.17457 & -0.29 & \\
\hline & -3.488176 & -1.200 & -3.4 \\
\hline & & & \\
\hline $\mathrm{H}$ & -4.08046 & 349 & \\
\hline & 3.217299 & 0.396 & -1.0 \\
\hline C & 4.02 & & -0.1 \\
\hline & 4.94 & 2.024 & -0.60 \\
\hline $\mathrm{H}$ & 5.56 & 2.54 & \\
\hline $\mathrm{C}$ & 5.06 & 2.31 & -1.9 \\
\hline $\mathrm{H}$ & 5.78 & 3.06 & -2.2 \\
\hline $\mathrm{C}$ & 4.2802436 & 1.630 & -2.88 \\
\hline $\mathrm{H}$ & 4.3937658 & 1.85276 & -3.9 \\
\hline$y$ & 3.34589 & 0.664 & $-2.4^{\prime}$ \\
\hline C & 2.527573 & 84909844 & -3.5 \\
\hline $\mathrm{H}$ & 1.7887210 & -0.690 & 2425 \\
\hline$y$ & 3.40873053703141 & -1.02060 & -4.37 \\
\hline $\mathrm{H}$ & 4.14471408829389 & 55696339 & 51537 \\
\hline I & 2.78990193995274 & -1.60080 & -5.07 \\
\hline $\mathrm{H}$ & 3.95917530411342 & & \\
\hline $\mathrm{C}$ & 1.74861868851241 & 05070653 & 32958 \\
\hline $\mathrm{H}$ & 1.11151841665245 & 1.56774687984752 & -3.87 \\
\hline $\mathrm{H}$ & 1.10515128948410 & 0.32222967705938 & 20712 \\
\hline $\mathrm{H}$ & 2.42072932413830 & 1.50472829242611 & -5.068 \\
\hline $\mathrm{C}$ & 3.96587909027764 & 0.73812831988313 & 1.34912870973774 \\
\hline $\mathrm{H}$ & 3.19105693569177 & -0.02382031915958 & \\
\hline $\mathrm{C}$ & 3.55842036589787 & 1.96437200928804 & 2.18616405693598 \\
\hline $\mathrm{H}$ & 4.31902522366548 & 2.75658140879660 & 2.14525787298944 \\
\hline
\end{tabular}




\begin{tabular}{|c|c|c|c|}
\hline & 3.42971146266259 & 1.68052358851500 & \\
\hline & 2.61780296688893 & 2.39775321354433 & \\
\hline & 5.29840044152559 & 0.14469424007860 & \\
\hline & 5.60214178289652 & -0.72316219743010 & \\
\hline & 5.20887285468503 & & \\
\hline & 6.11090976173287 & 0.88262671553996 & 882 \\
\hline & 0.16162381708203 & -0.85348842585735 & 3.042250 \\
\hline & -0.49543726371158 & 0254 & 9534 \\
\hline & -0.79824183625922 & -2.4 & 90624 \\
\hline & -0.78069287058319 & 46073593191 & 4.07456 \\
\hline & -1.30308880067193 & & \\
\hline & -0.42866145813419 & -2.4338488730 & 0085 \\
\hline & 0.24 & -1.203 & \\
\hline & 0.550 & -0.83 & 50 \\
\hline & 0.53093841078094 & -0.43291450 & 4.3 \\
\hline & 1.043 & & \\
\hline & -0.75133640995624 & -3.2 & 235 \\
\hline & 5722386446 & -4.0 & 6.3 \\
\hline & 258542 & -2.6 & 6512 \\
\hline & 887427 & -3.6 & \\
\hline & 0553997605 & 3.0 & -0.8 \\
\hline & 0.914 & & -1.6 \\
\hline & 1.824 & & -1.9 \\
\hline & 7422468255 & 5.0 & -2.0 \\
\hline $\mathrm{H}$ & 1.396 & & -2.6 \\
\hline & 9284098740 & 5.7467230038 & -1.6 \\
\hline C & 3642697712 & & -0.7 \\
\hline & 8041041323 & 5.56 & -0.4 \\
\hline & 0900994263 & 8289 & -0.38246 \\
\hline & 54184177908 & & 0.25390 \\
\hline 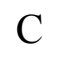 & -0.80 & & \\
\hline $\mathrm{H}$ & 24718042667 & 264 & 74638 \\
\hline $\mathrm{H}$ & 0.110 & 7.6 & -2.44 \\
\hline $\mathrm{H}$ & -1.25599117438317 & 7.75973811191661 & -1.31502 \\
\hline $\mathrm{N}$ & 2.23637755223985 & -0.56894975420137 & -0.655531 \\
\hline $\mathrm{N}$ & 0.23617429447678 & -2.05709291311544 & -0.39475457286226 \\
\hline & -1.76333720853356 & -0.55383874669535 & -0.403486 \\
\hline $\mathrm{N}$ & 0.42283017287998 & -0.03714159084172 & 1.95461705333130 \\
\hline & 0.20105277607541 & 1.76154452188386 & -0.42368021311479 \\
\hline & 0.27873558581694 & 0.00284847974372 & -0.07437987243476 \\
\hline & 0.79820281397827 & 0.84947668436066 & 2.27108559732127 \\
\hline
\end{tabular}

